\title{
Oesophageal submucosal hematoma after flow diverter embolization with favourable outcome treated by discontinuing postoperative antiplatelet therapy for only three days: a case report
}

\author{
Eriko Takeyama ${ }^{1}$, Aiko Wada², Eizo Amano ${ }^{1}$ and Hiromi Shibuya ${ }^{1 *}$
}

\begin{abstract}
Background: Oesophageal submucosal hematoma is a rare perioperative complication. When this complication develops after endovascular surgery, which requires postoperative antiplatelet therapy, whether to stop antiplatelet therapy or not is controversial. If antiplatelet therapy is discontinued, the appropriate time to resume antiplatelet therapy is unclear.

Case presentation: A 75-year-old woman (height $134 \mathrm{~cm}$, weight $37 \mathrm{~kg}$ ) underwent flow diverter embolization for unruptured cerebral aneurysm under general anaesthesia. The patient received dual antiplatelet therapy before surgery and anticoagulation therapy intraoperatively. After surgery, the patient developed hematemesis and was diagnosed with oesophageal submucosal hematoma. Conservative treatment was initiated after discontinuing antiplatelet therapy, which was resumed 3 days after surgery. The patient showed good recovery even after the resumption of antiplatelet therapy.
\end{abstract}

Conclusions: In our case, we successfully treated oesophageal submucosal hematoma developing after endovascular surgery with early resumption of postoperative antiplatelet therapy.

Keywords: Oesophageal submucosal hematoma, Endovascular surgery, Antiplatelet therapy

\section{Background}

Oesophageal submucosal hematoma is a rare perioperative complication. There have been several reports of this complication after endovascular surgery, which requires perioperative antiplatelet therapy. For these cases, physicians have to treat patients considering both the management of haemorrhage associated with the oesophageal submucosal hematoma and the prevention of thromboembolic complications after endovascular treatment. We report a case of oesophageal submucosal hematoma after placement of a flow diverter, a new-generation endovascular device for unruptured cerebral aneurysm-associated

\footnotetext{
* Correspondence: shibuya.hiromi.np@mail.hosp.go.jp

'Department of Anesthesiology, National Hospital Organization Osaka National Hospital, 2-1-14, Hoenzaka, Chuo-Ku, Osaka-city, Osaka 540-0006, Japan

Full list of author information is available at the end of the article
}

long-term postoperative antiplatelet therapy to prevent thromboembolic complications. We treated the patient successfully by suspending the antiplatelet therapy temporally and resuming it 3 days after hematoma development. To the best of our knowledge, this is the first report of a patient with oesophageal submucosal hematoma resuming antiplatelet therapy after only 3 days of suspension.

\section{Case presentation}

A 75-year-old woman (height $134 \mathrm{~cm}$, weight $37 \mathrm{~kg}$ ) underwent flow diverter placement for an unruptured cerebral aneurysm under general anaesthesia. Her medical history and preoperative complications were unremarkable. Preoperative laboratory data were within normal limits, with no coagulation abnormalities. She 
received dual antiplatelet therapy with aspirin and clopidogrel beginning 10 days prior to surgery.

Anaesthesia was induced with a target-controlled infusion of propofol $(3.0 \mu \mathrm{g} / \mathrm{ml})$ and fentanyl $(50 \mu \mathrm{g})$. After administration of rocuronium (30 mg), tracheal intubation was performed uneventfully with a video laryngoscope (McGRATH MAC ${ }^{\mathrm{mn}}$ ), followed by smooth insertion of a gastric tube. The gastric tube was maintained without suction intraoperatively. Anaesthesia was maintained with target-controlled infusion of propofol and remifentanil $0.05-0.1 \mu \mathrm{g} / \mathrm{kg} / \mathrm{min}$ with an inhaled oxygen concentration of $42 \%$ under standard monitoring, as well as direct radial artery pressure monitoring.

Heparin (4000 units) was intravenously administered at the beginning of surgery. Activating clotting time was 159, 254, and $244 \mathrm{~s}$ before surgery, $15 \mathrm{~min}$ after starting surgery, and at the end of surgery, respectively. The operative approach was from the femoral artery. Surgery was performed without complications. Protamine was not administered at the end of surgery. Intraoperatively, the patients' blood pressure remained at 80-120/40-50 $\mathrm{mmHg}$ and her heart rate remained at $55-65$ beats/min with no acute hemodynamic changes.

Continuous infusion of propofol and remifentanil were discontinued at the completion of surgery. The patient awakened $20 \mathrm{~min}$ after completion of surgery, and her systolic blood pressure increased to $150 \mathrm{mmHg}$. The gastric tube was removed without any abnormal findings, such as blood in the secretions. The tracheal tube was removed smoothly. Continuous infusion of argatroban was initiated at completion of surgery. The operation time was $97 \mathrm{~min}$, and anaesthesia time was 172 $\min$.

Due to systolic blood pressure greater than 150 $\mathrm{mmHg}$, continuous infusion of nicardipine was initiated to maintain systolic pressure below $140 \mathrm{mmHg}$ after entering the recovery room. The patient started to complain of chest pain $90 \mathrm{~min}$ after entering the recovery room. At that time, her blood pressure was 155/77 $\mathrm{mmHg}$ and her pulse rate was approximately $70 \mathrm{bpm}$. The electrocardiogram was unremarkable, and blood biochemical haemostatic function testing showed no abnormal findings without prolonged activated partial thromboplastin time $(120 \mathrm{~s})$. She developed hematemesis $120 \mathrm{~min}$ after entering the recovery room. Her blood pressure and heart rate remained stable. Emergent computed tomography $(\mathrm{CT})$ was performed. Although the vascular system was normal, expansion of the oesophagus was noted (Fig. 1). Due to a decrease in blood pressure to $90 / 47 \mathrm{mmHg}$ and haemoglobin level from 11.7 to $8.7 \mathrm{~g} / \mathrm{dL}$, the patient was given fluid resuscitation and was transfused with 6 units of red blood cells and 6 units of fresh frozen plasma following CT examination. Considering the risk of tracheal aspiration, we

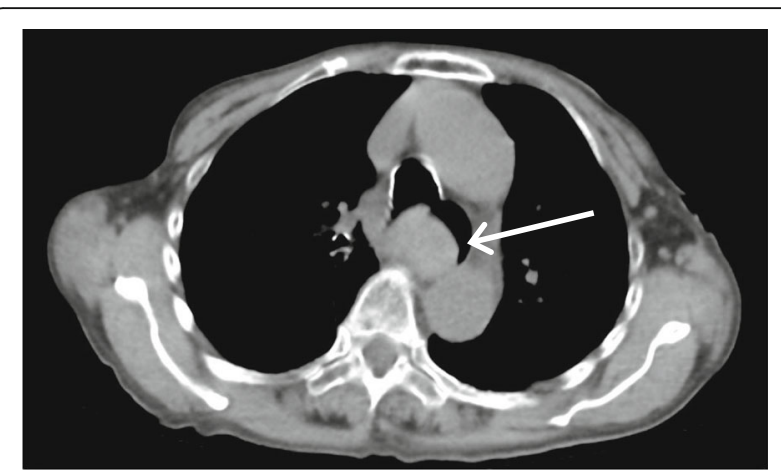

Fig. 1 Computed tomography thoracic examination immediately after hematemesis. The thoracic oesophagus is dilated. The white arrow indicates the oesophagus

performed tracheal intubation. Upper gastrointestinal endoscopy (UGE) was performed, and an oesophageal submucosal hematoma extending longitudinally on the right wall, $17 \mathrm{~cm}$ from the incisor, was noted. Because a narrowed lumen and oozing due to scope contact were recognised, observation was limited to the lower oesophagus (Fig. 2).

Conservative treatment was initiated with the cessation of continuous infusion of argatroban and the planned postoperative antiplatelet therapy. CT performed 2 days postoperatively showed reduced dilation of the oesophagus, and thus, the tracheal tube was removed. A nasogastric tube was inserted 3 days after surgery without any abnormal findings, and antiplatelet therapy was resumed on the same day. The patient had no symptoms even after the resumption of antiplatelet therapy. UGE performed 11 days postoperatively showed that the submucosal hematoma was almost completely resolved, and solid food intake was resumed. The patient showed good recovery and was discharged 27 days after surgery.

\section{Discussion}

Oesophageal submucosal hematoma is a rare condition. The spontaneous type of oesophageal submucosal hematoma is considered to be caused by the rupture of blood vessels in the submucosal layer [1], as the result of a sudden increase in pressure induced by factors such as nausea and vomiting. It is also suggested that oesophageal submucosal hematoma is associated with bleeding tendency, such as antiplatelet or anticoagulant therapy [2]. Although reports of intraoperative oesophageal submucosal hematoma are rare, a few cases have been reported of this complication after endovascular surgery for unruptured cerebral aneurysms. Endovascular surgery requires perioperative antiplatelet therapy, which could be associated with the development of oesophageal submucosal hematoma. The prognosis of this complication is generally 

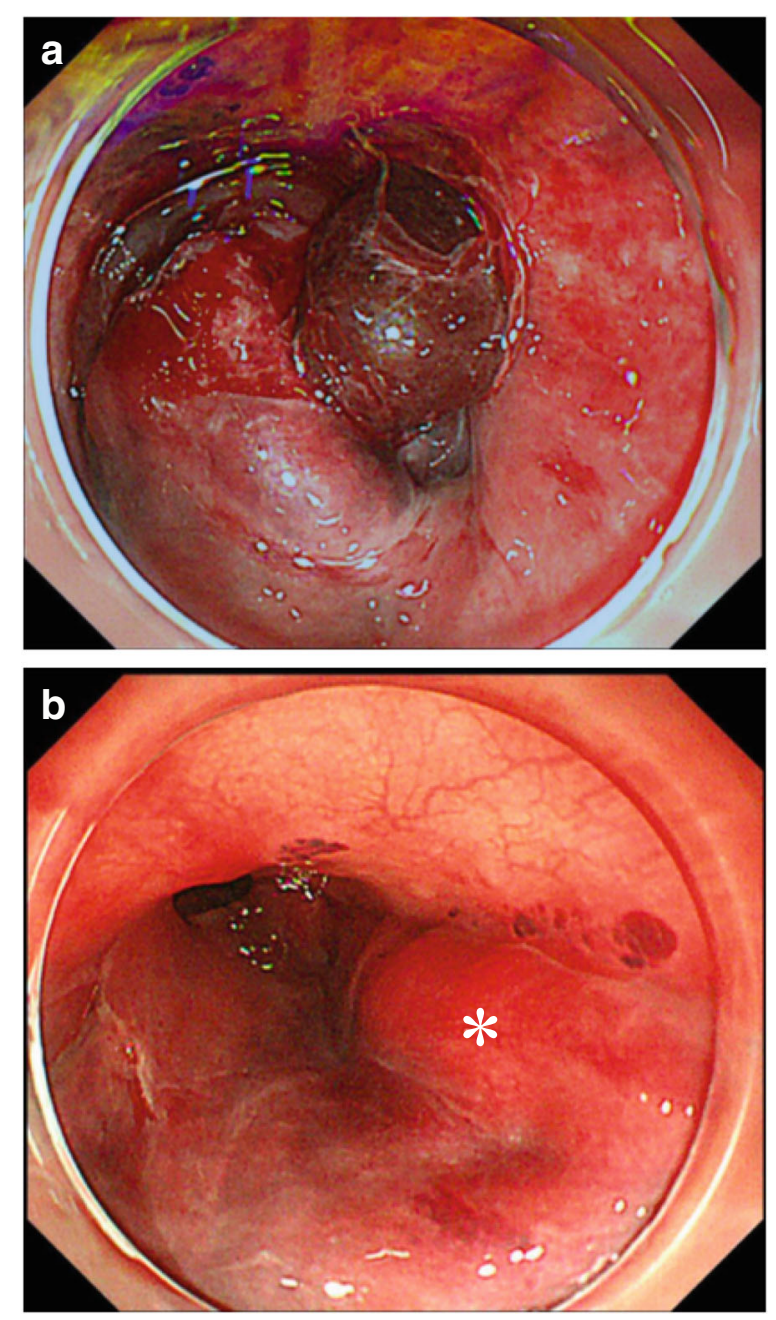

Fig. 2 Upper gastrointestinal endoscopy images after hematemesis. a The submucosal hematoma occupied the oesophagus. $\mathbf{b}$, a Longitudinal extension of reddish or wine-coloured mucosal thickening (asterisk), obstructing the oesophagus, is seen from the cervical oesophagus to the thoracic oesophagus

good, and approximately $90 \%$ of reported cases receiving antiplatelet therapy had been treated conservatively with cessation of postoperative antiplatelet therapy [3-9].

The flow diverter is a new-generation stent placed in the parent artery at the level of the aneurysm neck to disrupt inflow into the aneurysm sac and, thus, favour intra-aneurysm thrombosis. It is clinically used for large cerebral aneurysms that are difficult to treat with coil embolization. Conversely, long-term postoperative antiplatelet therapy is necessary to prevent severe thromboembolic complications, since a prolonged time period is required for aneurysm resolution. However, there are no clear criteria for drug selection, administration period, and short-term discontinuation of antiplatelet therapy due to haemorrhagic events.

Therefore, oesophageal submucosal hematoma after flow diverter placement must be treated considering not only the risk of bleeding but also the possibility of thromboembolic complications.

Considering the risk of bleeding, the risk of potentially severe oesophageal submucosal hematoma should be considered. Although this complication often shows a good prognosis, cases of haemorrhagic shock associated with the onset of oesophageal submucosal hematoma have been reported [4]. In our case, the patient developed massive bleeding requiring transfusion. As the guideline for flow diverter use recommends cessation of antiplatelet therapy when a severe bleeding event occurs, discontinuation of antiplatelet therapy immediately after the development of this complication can be considered reasonable.

After cessation of antiplatelet therapy, the optimum timing to resume treatment is a serious concern due to the possibility of thromboembolic complications. While there are no broadly accepted practice patterns addressing dual antiplatelet therapy in the flow diversion literature, the length and dose of antiplatelet therapy are reported to correlate with a decreased incidence of thrombotic and haemorrhagic events after flow diverter placement [10]. In our case, we diagnosed haemostasis at a relatively early stage by CT examination and resumed treatment 3 days after surgery. Even after the resumption of antiplatelet therapy, the patient did not show relapse of symptoms and had a favourable course. In almost every reported case treated conservatively, hematoma contraction has been confirmed by $\mathrm{CT}$ or upper gastrointestinal endoscopy within 4 to 7 days after onset. These reports may suggest that active bleeding stops at this time point and that resumption of antiplatelet therapy may be considered.

In conclusion, the risk of oesophageal submucosal hematoma development after endovascular surgery should be considered. When postoperative antiplatelet therapy is necessary, such as flow diversion, it may be beneficial to perform periodic reassessments for haemostasis or oesophageal submucosal hematoma, and early resumption of antiplatelet therapy may be considered with careful observation.

\section{Abbreviations}

CT: Computed tomography; UGE: Upper gastrointestinal endoscopy

\section{Acknowledgements \\ None.}

\section{Funding}

This research did not receive any specific grant from agencies in the public, commercial, or not-for profit sectors.

Availability of data and materials

The datasets analysed in this article are included within article. 


\section{Authors' contributions}

AW and HS provided anaesthetic care. ET and EA drafted the manuscript. All authors revised the manuscript critically and approved the final version for publication.

\section{Ethics approval and consent to participate}

Not applicable.

\section{Consent for publication}

Written informed consent was obtained from the patient for publication of this case report and accompanying images.

\section{Competing interests}

The authors declare that they have no competing interests.

\section{Publisher's Note}

Springer Nature remains neutral with regard to jurisdictional claims in published maps and institutional affiliations.

\section{Author details}

'Department of Anesthesiology, National Hospital Organization Osaka National Hospital, 2-1-14, Hoenzaka, Chuo-Ku, Osaka-city, Osaka 540-0006, Japan. ${ }^{2}$ Department of Anesthesiology and Intensive Care Medicine, Graduate School of Medicine, Osaka University, 2-2, Yamada-oka, Suita, Osaka 565-0871, Japan.

Received: 6 December 2018 Accepted: 26 December 2018

Published online: 08 January 2019

\section{References}

1. Freeman AH, Dickinson RJ. Spontaneous intramural oesophageal haematoma. Clin Radiol. 1988;39:628-34.

2. Hong M, Warum D, Karamanian A. Spontaneous intramural esophageal hematoma (IEH) secondary to anticoagulation and/or thrombolysis therapy in the setting of a pulmonary embolism: a case report. J Radiol Case Rep. 2013;7:1-10.

3. Yamashita K, Okuda H, Fukushima H, Arimura Y, Endo T, Imai K. A case of intramural esophageal hematoma: complication of anticoagulation with heparin. Gastrointest Endosc. 2000;52:559-61.

4. Ito S, Iwata S, Kondo I, Iwade M, Ozaki M, Ishikawa T, et al. Esophageal submucosal hematoma developed after endovascular surgery for unruptured cerebral aneurysm under general anesthesia: a case report. JA Clin Rep. 2017;3:54

5. Fujimoto Y, Shirozu K, Shirozu N, Akiyoshi K, Nishimura A, Kawasaki S, et al. Esophageal submucosal hematoma possibly caused by gastric tube insertion under general anesthesia. A A Case Rep. 2016;7:169-71.

6. Chiu YH, Chen JD, Hsu CY, How CK, Yen DH, Huang Cl. Spontaneous esophageal injury: esophageal intramural hematoma. J Chin Med Assoc. 2009;72:498-500.

7. Oe S, Watanabe K, Kume K, Shibata M, Hiura M, Yoshikawa I, Harada M. A case of idiopathic gastroesophageal submucosal hematoma and its disappearance observed by endoscopy. JUEOH. 2014;1:123-8.

8. Saito S, Hosoya Y, Kurashina K, Yokoyama T, Arai W, Hyodo M. Esophageal submucosal hematoma: a case report and review of the literature. Esophagus. 2005;2(3):155-9.

9. Polat C, Dervisoglu A, Guven H, Kaya E, Malazgirt Z, Danaci M, Ozkan K. Anticoagulant-induced intramural intestinal hematoma. Am J Emerg Med. 2003;21:208-11.

10. Skukalek SL, Winkler AM, Kang J, Dion JE, Cawley CM, Webb A, et al. Effect of antiplatelet therapy and platelet function testing on hemorrhagic and thrombotic complications in patients with cerebral aneurysms treated with the pipeline embolization device: a review and meta-analysis. J Neurointerv Surg. 2016:8:58-65.

\section{Submit your manuscript to a SpringerOpen ${ }^{\circ}$ journal and benefit from:}

- Convenient online submission

- Rigorous peer review

- Open access: articles freely available online

- High visibility within the field

- Retaining the copyright to your article

Submit your next manuscript at $\boldsymbol{\nabla}$ springeropen.com 